\title{
Topological susceptibility with the improved Asqtad action
}

\author{
MILC Collaboration: C. Bernard ${ }^{\text {a }}$, T. Burch ${ }^{\text {b }}$, T.A. DeGrand ${ }^{\text {c}}$, C.E. DeTar ${ }^{\text {d* }}$, Steven Gottlieb ${ }^{\text {e, }}$ \\ E. Gregory ${ }^{b}$, A. Hasenfratz ${ }^{\text {c }}$, U.M. Heller ${ }^{f}$, J. Hetrick ${ }^{g}$, J. Osborn ${ }^{d}$, R.L. Sugar ${ }^{\text {h }}$, and D. Toussaint ${ }^{b}$ \\ ${ }^{a}$ Department of Physics, Washington University, St. Louis, MO 63130, USA \\ ${ }^{\mathrm{b}}$ Department of Physics, University of Arizona, Tucson, AZ 85721, USA

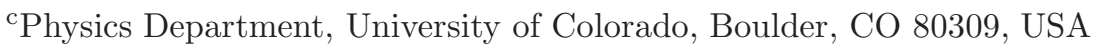 \\ ${ }^{\mathrm{d}}$ Physics Department, University of Utah, Salt Lake City, UT 84112, USA \\ e Department of Physics, Indiana University, Bloomington, IN 47405, USA and Fermilab, Batavia, IL \\ 60510, USA \\ ${ }^{\mathrm{f}}$ CSIT, Florida State University, Tallahassee, FL 32306-4120, USA \\ gUniversity of the Pacific, Stockton, CA 95211, USA \\ ${ }^{\mathrm{h}}$ Department of Physics, University of California, Santa Barbara, CA 93106, USA
}

As a test of the chiral properties of the improved Asqtad (staggered fermion) action, we have been measuring the topological susceptibility as a function of quark masses for $2+1$ dynamical flavors. We report preliminary results, which show reasonable agreement with leading order chiral perturbation theory for lattice spacing less than $0.1 \mathrm{fm}$. The total topological charge, however, shows strong persistence over Monte Carlo time.

\section{INTRODUCTION}

The cost of lattice simulations increases dramatically as the lattice spacing is decreased. Improvement programs seek to avoid these costs by reducing lattice artifacts at larger lattice spacing. Whether lattice artifacts are successfully reduced depends on the observable. The staggered fermion Asqtad action [1] is designed to remove $\mathcal{O}\left(a^{2}\right)$ lattice artifacts at tree level, resulting in an improved flavor symmetry and better chiral properties [1,2]. However, the Asqtad quarkgluon vertex is not as smooth as that of the more elaborate HYP action [3] and zero modes are not treated as rigorously as with the more expensive domain wall and overlap actions. Through a rougher vertex, quark propagation might be influenced by small instanton-like dislocations, and with imprecise zero modes, at small quark mass the fermion determinant may fail to suppress ad-

\footnotetext{
*Presented by C. DeTar.
}

equately configurations with nonzero topological charge.

\section{TOPOLOGICAL SUSCEPTIBILITY}

A good test of the effectiveness of zero mode suppression is the identity in chiral perturbation theory [4] relating the susceptibility of the topological charge to the light quark masses

$\chi_{\text {top }}=\frac{\left\langle Q^{2}\right\rangle}{V}=\frac{\Sigma}{\left(\frac{1}{m_{u}}+\frac{1}{m_{d}}+\frac{1}{m_{s}}\right)}$,

where $Q=\int F_{\mu \nu}^{a} \tilde{F}_{\mu \nu}^{a} d^{4} x / 32 \pi^{2}$. For three flavors with $m_{u}=m_{d}$ we write the susceptibility as

$\chi_{\mathrm{top}}=f_{\pi}^{2} m_{\pi}^{2} /\left[4\left(1+m_{u d} / 2 m_{s}\right)\right]$,

showing that it vanishes linearly in the square of the pion mass in the chiral limit.

Shown in Figs. 1 and 2 are recent results for the $N_{f}=2$ relation, for the unimproved and improved Wilson actions and the conventional thin- 


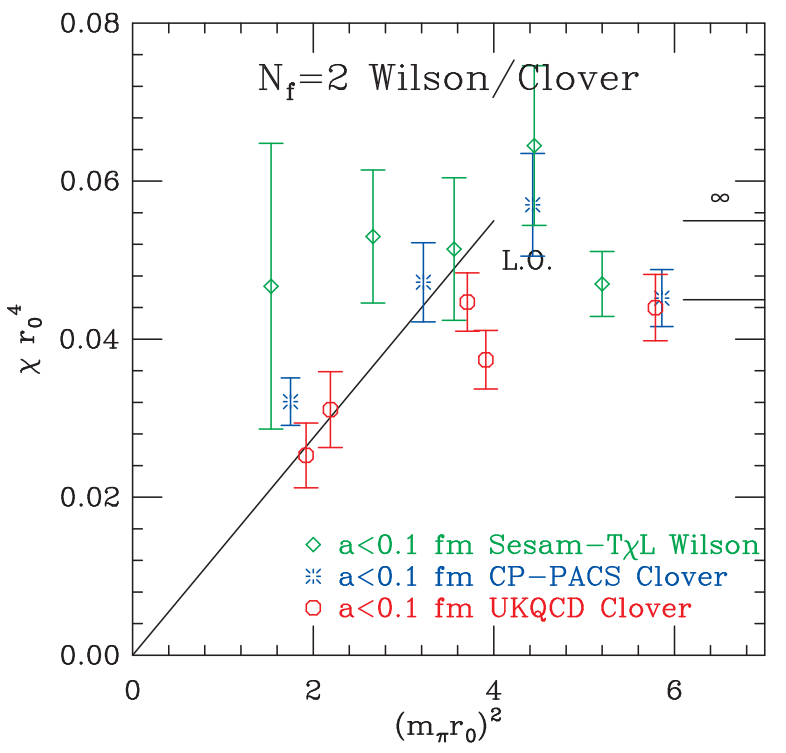

Figure 1. Topological susceptibility for the unimproved and improved Wilson action vs. pion mass squared in units of $r_{0}$ 5. Lines at the right indicate the quenched value.

link staggered fermion action [3,6,6], suggesting that at a lattice spacing $a<0.1 \mathrm{fm}$ the chiral prediction 2 is reasonably well satisfied as far as testing was possible. More recently, as shown in Fig. 2 the relation was tested in simulations with dynamical HYP fermions at $a=0.17 \mathrm{fm}$ with a dramatic improvement over thin-link, but suggesting that even here a smaller lattice spacing is needed [7].

\section{MEASUREMENT}

We have measured the topological charge on an ensemble of $20^{3} \times 64(a=0.13 \mathrm{fm})$ and $28^{3} \times 96$ $(a=0.09 \mathrm{fm})$ gauge configurations generated in the presence of $2+1$ flavors of Asqtad dynamical quarks of varying masses. The charge is obtained by integrating the topological charge density, defined as an approximation to $F_{\mu \nu}^{a} \tilde{F}_{\mu \nu}^{a} / 32 \pi^{2}$. As usual, prior to measuring the topological charge, it is necessary to smooth the gauge configurations to remove short wavelength fluctuations. Smoothing was done with a series of hypercubic blocking sweeps [8]. This method seeks to achieve

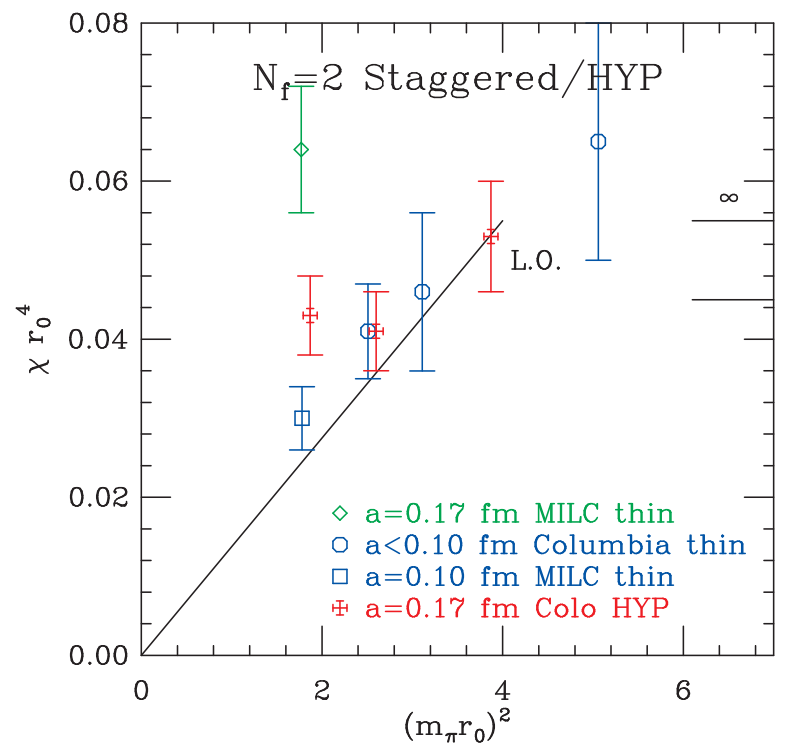

Figure 2. Topological susceptibility for the unimproved thin-link and improved HYP staggered fermion action vs. pion mass squared in units of $r_{0}$ 6.7. L. Lines at the right indicate the quenched value.

a smooth configuration with a minimal distortion of the topological structures [6]. Accordingly we take as few smoothing sweeps as necessary, and find that the susceptibility is constant within errors after from one to six such sweeps. The improved cooling technique gave similar values of $Q$ [9].

Results are shown in Fig. 3. Evidently, the Asqtad action, like the conventional staggered action, requires a lattice spacing less than approximately $0.1 \mathrm{fm}$ to obtain reasonably good agreement with leading order chiral perturbation theory.

\section{PERSISTENCE OF TOPOLOGICAL CHARGE}

A particularly noteworthy feature of our measurements is the persistence of the total topological charge. Time histories for two light quark masses are shown in Fig. 1. This phenomenon has been noted for the conventional staggered fermion action 10 and appears to be rather more pronounced for Asqtad with a better gauge action at 


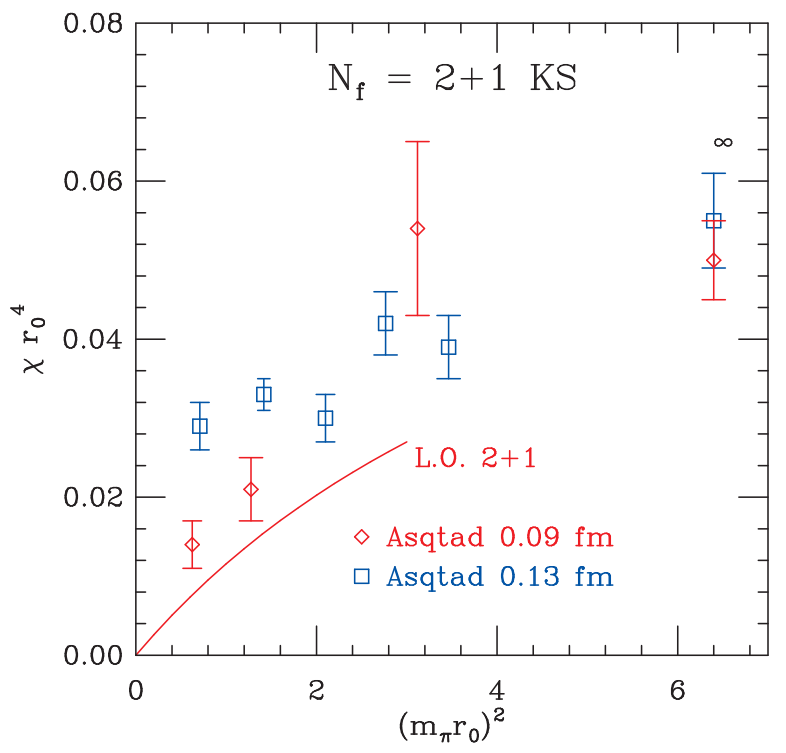

Figure 3. Topological susceptibility vs. pion mass squared for the Asqtad action with $2+1$ flavors. The curve gives the prediction of leading order chiral perturbation theory. Quenched values are plotted on the extreme right.

comparable lattice spacing and pion mass, but in our case worse for the heavier light quark. In both cases the $\mathrm{R}$ algorithm is used in the updating process. For the pure gauge theory, overrelaxation plus heatbath updating decorrelates rapidly.

\section{CONCLUSIONS}

Our preliminary findings are that the Asqtad action, like the conventional staggered fermion action, requires a lattice spacing less than approximately $0.1 \mathrm{fm}$ to give reasonably good chiral behavior for the topological susceptibility. However, in connection with a small step-size updating algorithm, the action gives strong persistence of the topological charge. We are investigating the implications of these results.

Computations were performed at LANL, NERSC, NCSA, ORNL, PSC, SDSC, FNAL, the CHPC (Utah) and the Indiana University SP. This work is supported by the U.S. NSF and DOE.

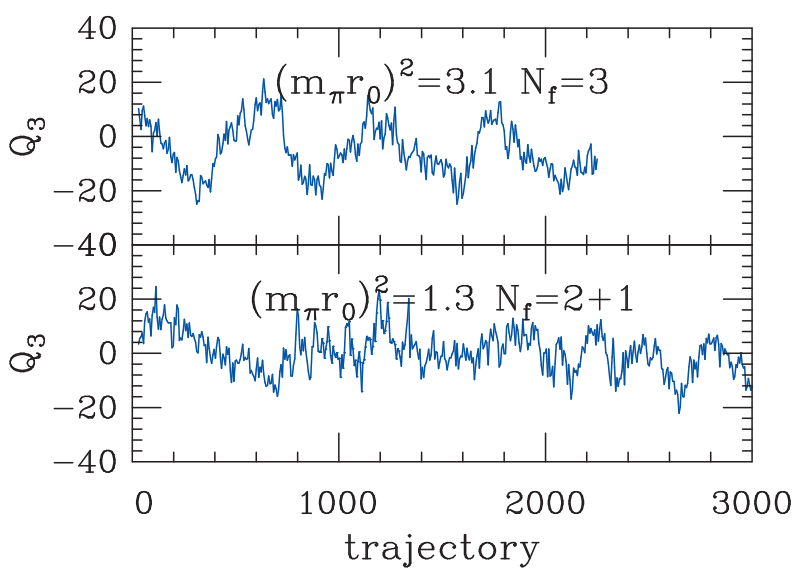

Figure 4. Time history of topological charge after three HYP sweeps vs. trajectory at two values of $m_{u, d}$ in the $a=0.09 \mathrm{fm}$ Asqtad simulation.

\section{REFERENCES}

1. K. Orginos and D. Toussaint, Phys. Rev. D 59 (1999) 014501; K. Orginos, D. Toussaint and R. L. Sugar, Phys. Rev. D 60 (1999) 054503; G. P. Lepage, Phys. Rev. D 59 (1999) 074502 .

2. C. Bernard et al., Phys. Rev. D 64 (2001) 054506 .

3. F. Knechtli and A. Hasenfratz, Phys. Rev. D 63 (2001) 114502 and hep-lat/020301d.

4. H. Leutwyler and A. Smilga, Phys. Rev. D 46 (1992) 5607.

5. G. S. Bali et al. $[\mathrm{T} \chi \mathrm{L}$ Collaboration], Phys. Rev. D 64 (2001) 054502. A. Ali Khan et al. [CP-PACS Collaboration], Phys. Rev. D 64 (2001) 114501. A. Hart and M. Teper [UKQCD Collaboration], Phys. Lett. B $5 \mathbf{2 3}$ (2001) 280.

6. A. Hasenfratz, Phys. Rev. D 64 (2001) 074503 .

7. A. Hasenfratz, this conference (2002).

8. A. Hasenfratz and F. Knechtli, Phys. Rev. D 64 (2001) 034504.

9. J. Hetrick, this conference (2002).

10. G. Boyd, B. Alles, M. D'Elia and A. Di Giacomo, hep-lat/9711025. 